DOI: $10.21554 / \mathrm{hrr} .091809$

\title{
VISUAL PERCEPTION OF SPEECH IN CHILDREN WITH COCHLEAR IMPLANT
}

\author{
Azra Hilić-Huskić ${ }^{1}$ \\ Esad H. Mahmutović \\ Meliha Povlakić Hadžiefendić
}

Original scientific paper

Association „, We exist too“, Velika Kladuša, Bosnia and Herzegovina

Center for Education and Rehabilitation of hearing and speaking Tuzla, Bosnia and Herzegovina

Center for Hearing and Speech Rehabilitation, Sarajevo, Bosnia and Herzegovina

\begin{abstract}
By the development and application of cochlear implants, a large number of people with hearing impairment realize a better perception of speech after implantation. The aim of the research was to determine the differences in the quality of the perception of speech of children with cochlear implant in relation to the perception modality (auditory, visual, and audiovisual). The sample consisted of 30 deaf children with a cochlear implant, both sexes, chronologically aged from 3 to 15 years old, who regularly attend or had attended rehabilitation of hearing and speaking. The Test Lingvogram and the Articulation Test were used for testing (Vuletic, 1990). The data were processed with descriptive statistics and singlefactor analysis of variance. Respondents had the weakest results of word repetition and word understanding in the visual modality, much better results in auditory modality, and the best results in audiovisual modality. By comparing different modalities of speech perception, it was found that the differences were statistically significant in all pairs of modalities, both in word repetition and in word understanding, at the level of statistical significance $p<.05$, except between the visual and auditory perception ( $p=.26$ ) in word repetition, but they were clinically significant in this combination too. The reason for the better effects of the modalities of the auditory and especially audiovisual perception, in relation to the visual perception of speech in this study is the application of cochlear implants in improving hearing and listening. However, people with a cochlear implant are still persons with hearing impairment. They should always have a high level of quality of the visual perception of speech in communication, which can be achieved by special exercises in the process of early rehabilitation of hearing and speaking.
\end{abstract}

Keywords: hearing impairment, cochlear implant, visual perception

\footnotetext{
${ }^{1}$ Correspodence to:

Azra Hilić-Huskić, Association „We exist too“, Velika Kladuša, Bosnia and Herzegovina

Ibrahima Mrzljaka 34, Velika Kladuša, Bosnia and Herzegovina

Phone:+387 62-405 401

E-mail: hilic.hilic@yahoo.com
} 


\section{INTRODUCTION}

Speechreading involves the visual channel of speech communication. It is a natural means of communication that we all use to supplement hearing, especially when listening conditions become difficult. It should thus come as no surprise that all people with hearing impairments must rely on speechreading to a greater or lesser extent (Ross, Brackett, \& Maxon 1982, see Gelfand, 2016, p. 433). Speechreading as used here means using the visual clues of the speaker's lip and facial movements, gestures, posture and body language, along with residual hearing to make use of the speaker's verbal communication, intonation and context to infer meaning (formerly known as lip reading). Speechreading is used by persons with typical hearing and those with hearing loss, especially when there are communication challenges such as background noise, and is an integral part of speech processing (,Speechreading“, 2016).

By people with a standard hearing the role of visual perception, of the voice signal in understanding the voice message in the noise, in reverberation, and even in situations where the speech signal can be heard well, but is difficult to understand because of linguistic or content complexity, is more significant than is usually thought. In cases where, due to noise or reverberation, the voice signal cannot be heard well, the possibility of its visual perception, with dominant audible perception, significantly improves the understanding of speech of people with standard hearing (Bradarić-Jončić, 1997).

The role of hearing and speech rehabilitation for a deaf child is extremely important. It is very important to raise the ability of visual speech perception through exercises carried out by a well-trained expert. If the child carrying the device is found in conditions of hearing deprivation, e.g. due to technical problems or problems of some other kind, it will always have good support for visual perception, developed through these exercises.

By the development and application of cochlear implants, a large number of people with hearing impairment realize a better perception of speech after implantation.

According to Spencer (2016, p. 13), data about language development using CIs have become available on larger numbers of children (Nicholas \& Geers, 2007, 2008; Niparko et al., 2010), and outcomes are more positive as technology improves and age of implantation decreases. A number of factors beyond age of implantation have been implicated in spoken language outcomes (e.g., Niparko et al., 2010; Nittrouer,
2010; Spencer, 2004), and reports continue of great variability (e.g., Tobey et al., 2013). However, other characteristics and experiences being roughly equal, it has been well established that children with early use of CIs generally outperform those with the same degree of hearing loss who use only hearing aids - or who did not use CIs until later ages. In fact, Tobey et al. reported a general consensus that children who receive CIs by 18 months of age develop better spoken language skills, on average, than those implanted later.

According to Kral (2013, p.7), recent studies indicate the superiority of outcomes of even earlier implantation (within the first 18 months; De Raeve, 2010; Niparko et al., 2010; Yoon, 2011). These results emphasize that the eventual goal should be to use prosthetic devices such as cochlear implants to provide children with hearing as early as possible. Effective communication before implantation has been shown to be a predictor of the success of postimplantation communication (Tait et al., 2000).

A summary measure of the advances being made in language development was provided by Goberis et al. (2012), according to Spencer (2016, p.13), who noted that DHH children with high-quality early intervention and without complications of limited cognitive abilities or significant multiple disabilities can develop language at a rate of about $80 \%$ to even $100 \%$ of that of hearing children. Previously, this rate (even in the best of conditions) was about $45 \%-65 \%$. In addition, evidence is accruing that, unlike for previous generations, the faster rate of development may be being maintained through older ages instead of leveling or even dropping at adolescence in some domains.

While cochlear implants work and appear to work well for many profoundly deaf adults and children, they do not always provide benefits to all patients who receive them. Compared to other behavioral data I have seen in the field of speech perception and spoken word recognition over the years, the audiological outcomes and benefits following cochlear implantation were simply enormous and hard to fully understand at first glance. Some deaf adults and children do extremely well with their cochlear implants and display what initially appears to be near-typical speech perception and language skills on a wide range of traditional clinical speech and language tests when tested under quiet listening conditions in the laboratory. In contrast, other adults and children struggle for long periods of time after they receive their cochlear implant and often never achieve comparable levels of speech and language performance or verbal fluency (Pisoni, 2004, p.134). 
During rehabilitation procedures, the visual perception of speech, regardless of the system of communication that is adopted, and especially if oral speech is adopted, requires a special approach.

Deaf and hard-of-hearing people should always have a high level of visual perception of speech in communication, regardless of chronological age, and this can be achieved by special exercises in the process of early rehabilitation of hearing and speaking. Methods that influence the better visual perception of speech, in the earlier period, before the appearance of cochlear implants, were extremely represented. With the installation of cochlear implants, it is noticeable that the visual perception of speech, especially the application of these methods, begins to be ignored.

The aim of the research was to determine the differences in the quality of the perception of speech of children with a cochlear implant compared to the modality of speech perception.

\section{WORK METHODS}

\section{Sample respondents}

The sample of respondents consisted of 30 deaf children with cochlear implant, both sexes, chronologically aged from 3 to 15 , who regularly attend or had attended rehabilitation of hearing and speaking.

\section{Measuring instrument}

In order to determine the quality of visual perception of speech, that is, the examination of elementary speech-linguistic abilities (direct repetition of the word - articulation of voices and word understanding), the modified and mutually combined Test Lingvogram (Kostić, Vladisavljević, Blagojević, 1983) and Articulation Test (Vuletić , 1990) were used.

The first part of the examination was conducted in such a way that the examiner followed the repetition of the word and the pronunciation of the examinees' voices when he names a certain term in the Lingvogram images, after perceiving the word from the examiner through three modalities of perception: auditory (the examiner covers the face while speaking), visual (the examinee removes the hearing instrument or the processor of the cochlear apparatus and lip-reads the speech of the examiner) and audiovisual (the examinee uses a hearing aid and looks at the examiners' lips). For each word, responses are recorded (exactly repeating words, substitution, distortion, and voice omis- sion), and then the summary result is entered in the appropriate form. Subsequently, the Articulation Test (Modified Test Form by Vuletić, 1990), from which the norms for assessing the articulation of voices were taken, was used to calculate the total damage of articulation (percentage value). Sample variables for this part of the test: word repetition and articulation damage.

The second part of the examination was carried out in such a way that the examiner names an image of a particular concept from the Lingvogram, and the examinee shows it, provided that the perception of the speech by the examinee, or the examination, also took place individually, through three different modalities (auditory, visual or audiovisual).

For each term or word, the response (correctly or incorrectly understanding the word) was recorded in the appropriate form and in the end the total number of responses was summed. The variable used to estimate the quality of visual perception of speech in this research was the word understanding.

\section{Data processing methods}

The data is processed in the SPSS for Windows program. The basic statistical parameters are calculated: the minimum and maximum results, the sum, the arithmetic mean, and the standard deviation. Single-factor variance analysis of repeat measurement (ANOVA) was applied in comparing data on the quality of perception in three different modalities (visual, auditory, audiovisual). For the multiple comparisons of data from different modalities of speech perception, the Bonferroni test for adaptation was used.

\section{RESULTS AND DISCUSSION}

Respondents had the weakest results of word repetition and word understanding in visual modality, much better results in auditory and best results in audiovisual modality. The average value of the repetition of the word perceived by the visual modality of perception was $38.07 \pm 23.00$, the audible $44.90 \pm 25.48$, and the audiovisual modality of $50.73 \pm 25.49$ words, of a total of 90 words.

Total articulation damage was on average $10.84 \pm$ 11.84.

The average value of word understanding perceived by the visual perception modality was $52.90 \pm 21.89$, by the auditory $62.17 \pm 19.68$, and by the audiovisual modality $63.97 \pm 19.31$ words, also, of a total of 90 words. 
Table 1. Basic statistical parameters

\begin{tabular}{lccccc}
\hline & N & MIN & MAX & M & SD \\
\hline Word repetition - visual & 30 & 5.00 & 88.00 & $\mathbf{3 8 . 0 7}$ & 23.00 \\
Word repetition - auditory & 30 & 12.00 & 90.00 & $\mathbf{4 4 . 9 0}$ & 25.48 \\
Word repetition - audiovisual & 30 & 12.00 & 90.00 & $\mathbf{5 0 . 7 3}$ & 25.49 \\
\hline Oštećenje artikulacije (\%) & 30 & .06 & 43.00 & $\mathbf{1 0 . 8 4}$ & 11.84 \\
\hline Word understanding - visual & 30 & .00 & 87.00 & $\mathbf{5 2 . 9 0}$ & 21.89 \\
Word understanding - auditory & 30 & 9.00 & 89.00 & $\mathbf{6 2 . 1 7}$ & 19.68 \\
Word understanding - audiovisual & 30 & 10.00 & 89.00 & $\mathbf{6 3 . 9 7}$ & 19.31 \\
\hline
\end{tabular}

The analysis of the variance of the repeated measurements determined a statistically significant difference between the group mean values and for the word repetition (Wilks' Lambda $=.57$; F (2.28) $=10.73$; Partial eta squared $=.43$ ) and for the word understanding $($ Wilks' Lambda $=.52 ; \mathrm{F}(2.28)=13.04$; Partial eta squared $=.48)$, at the level of statistical significance $p<.05$ (Table 2). Given the amounts of partial eta squares, it can be concluded that a statistically significant difference in the quality of speech perception is determined by the great influence of the different perception modalities.

Table 2. Multivariate Test

\begin{tabular}{lcccccc}
\hline & Wilks' Lambda & F & df & Error df & p & Partial Eta Squared \\
\hline Word repetition & .57 & 10.73 & 2 & 28.00 & .00 & .43 \\
Word understanding & .52 & 13.04 & 2 & 28.00 & .00 & .48 \\
\hline
\end{tabular}

With multiple comparison of data from different modalities of speech perception, with Bonferroni adjustment, it is possible to notice that differences are statistically significant in all modality pairs both in word repetition and in word understanding, at the level of statistical significance $p<.05$, except between visual and auditory perception $(\mathrm{p}=.26)$ of the word repetition, but are clinically significant in this combination too (Table 3).

Table 3. Pairwise Comparisons

\begin{tabular}{|c|c|c|c|c|}
\hline & Perception (I) & Perception (J) & Mean Difference (I-J) & $p$ \\
\hline \multirow{6}{*}{ Word repetition } & \multirow{2}{*}{ Visual } & Auditory & -6.83 & .26 \\
\hline & & Audiovisual & -12.67 & .00 \\
\hline & \multirow{2}{*}{ Auditory } & Visual & 6.83 & .26 \\
\hline & & AAudiovisual & -5.83 & .00 \\
\hline & \multirow{2}{*}{ Audiovisual } & Visual & 12.67 & .00 \\
\hline & & Auditory & 5.83 & .00 \\
\hline \multirow{6}{*}{ Word understanding } & \multirow{2}{*}{ Vizuelna } & Auditory & -9.27 & .02 \\
\hline & & Audiovisual & -11.07 & .00 \\
\hline & \multirow{2}{*}{ Auditory } & Visual & 9.27 & .02 \\
\hline & & Audiovisual & -1.80 & .00 \\
\hline & \multirow{2}{*}{ Audiovisual } & Visual & 11.07 & .00 \\
\hline & & Auditory & 1.80 & .00 \\
\hline
\end{tabular}


The reason for the better effects of the modalities of the auditory and especially audiovisual perception, in relation to the visual perception of speech in this study is the application of cochlear implants in improving hearing and listening, which cannot be achieved using classical hearing aids in subjects with this degree of hearing impairment.

According to Bergeson, Pisoni and Davis (2003, p. 348 ), in one of the first studies of AV speech perception in children with cochlear implants, Staller et al. (1991) administered the Word Intelligibility by Picture Identification (Lerman, Ross, \& McLauchlin, 1965) closed-set test of spoken word perception to 8-year-old children, and the Central Institute for the Deaf (Davis \& Silverman, 1978) open-set test of sentence perception to 12-year-old children. The children received cochlear implants at a mean age of 9.2 years and all children had used their cochlear implants for at least 1 year. Most, but not all, of the children were prelingually deafened. Children in both age groups performed better in the AV condition compared with a lipreading-alone (visual-alone, or $\mathrm{V}$-alone) condition, revealing that they benefited from the additional auditory information provided by their implant. However, because the investigators did not administer these tests in an auditory-alone (Aalone) condition, it is possible these children would have performed equally well in the AV condition and an A-alone condition.

They also state that more recent studies of AV speech perception in children with prelingual hearing loss who received cochlear implants at younger ages than children in the Staller et al. (1991) study have administered speech perception tests under three presentation conditions: A-alone, V-alone, and AV (Geers et al., 2003; Lachs et al., 2001; Tyler, Fryauf-Bertschy, et al., 1997). In the Tyler, Fryauf-Bertschy, et al. (1997) study, two separate groups of children completed the Audiovisual Feature Test (Tyler, Fryauf-Bertschy, \& Kelsay, 1991), a closed-set test of consonant feature recognition, at 2 and 4 years post-implantation. The results showed that performance was better in the AV presentation condition compared with the A-alone and $\mathrm{V}$-alone conditions, regardless of the consonant feature.

According to Geers, Brenner, and Davidson (2003), children, who received a cochlear implant under 5 $\mathrm{yr}$ of age, achieved an average level of about $40 \%$ speech recognition through lipreading alone, 50\% through listening alone and about $80 \%$ speech recognition through lipreading and listening together. Their auditory perception of speech features corresponded to $80 \%$ correct perception of vowels and $60 \%$ correct perception of consonants, which is roughly equivalent to that of a severely hearingimpaired child using hearing aids (Boothroyd \& Eran, 1994).

Bergeson, Pisoni and Davis (2003, p.347) the investigated the development of audiovisual speech perception skills in children who are prelingually deaf and received cochlear implants. Thay analyzed results from the Pediatric Speech Intelligibility (Jerger, Lewis, Hawkins, \& Jerger, 1980) test of audiovisual spoken word and sentence recognition skills obtained from a large group of young children with cochlear implants enrolled in a longitudinal study, from pre-implantation to 3 years post-implantation. The results revealed better performance under the audiovisual presentation condition compared with auditory-alone and visual-alone conditions. Performance in all three conditions improved over time following implantation. The results also revealed differential effects of early sensory and linguistic experience. Children from oral communication (OC) education backgrounds performed better overall than children from total communication (TC backgrounds. Finally, children in the early-implanted group performed better than children in the late-implanted group in the auditoryalone presentation condition after 2 years of cochlear implant use, whereas children in the lateimplanted group performed better than children in the early-implanted group in the visual-alone condition. The results of the present study suggest that measures of audiovisual speech perception may provide new methods to assess hearing, speech, and language development in young children with cochlear implants. The relationships observed between auditory-alone speech perception, audiovisual benefit, and speech intelligibility indicate that these abilities are not based on independent language skills, but instead reflect a common source of linguistic knowledge, used in both perception and production, that is based on the dynamic, articulatory motions of the vocal tract. The effects of communication mode demonstrate the important contribution of early sensory experience to perceptual development, specifically, language acquisition and the use of phonological processing skills. Intervention and treatment programs that aim to increase receptive and productive spoken language skills, therefore, may wish to emphasize the inherent cross-correlations that exist between auditory and visual sources of information in speech perception (Lachs, Pisoni, \& Kirk, 2001). 


\section{CONCLUSION}

By the development and application of cochlear implants, a large number of people with hearing impairment realize a better perception of speech after implantation.

However, the fact is that listening and hearing with hearing aids is not a natural process. People with a cochlear implant may suddenly, for various reasons (eg, the processor stops working), find themselves in conditions of hearing deprivation. They are thus still people with hearing impairment, and if there is no auditory perception, the ability of visual perception in communication then becomes significant.

Examination of its quality and role in the communication of children with cochlear implant is an important segment of monitoring and knowledge, both for providing adequate support in its development and as an important scientific and research issue.

This research showed that respondents had the weakest results of word repetition and word understanding precisely in visual modality, much better in auditory modality, and the best in audiovisual modality. A statistically significant difference was found in all pairs of perception modalities, both in word repetition and in word understanding, except between visual and auditory perception in the word repetition, but they were clinically significant in this combination too. Therefore, in the early development period, in addition to exercises for the development of auditory perception, intensive exercises are needed to improve the visual perception of speech as well.

\section{REFERENCES}

Bergeson, R.T., Pisoni, B.D. i Davis, A.O.R. (2003). A Longitudinal Study of Audiovisual Speech Perception by Children with Hearing Loss Who have Cochlear Implants. Volta Rev.103(4), pp. 347-370.

Bradarić-Jončić, S. (1997). Visual speech perception and deafness. Croatian review of rehabilitation research, 33 (2), pp. 119-131.

Geers, A., Brenner, C., \& Davidson, L. (2003). Factors associated with development of speech perception skills in children implanted by age five. Ear and Hearing, 24, pp. 24S$35 \mathrm{~S}$.

Gelfand, S. A. (2016). Essentials of audiology, fourth edition. New York: Thieme Medical Publishers, Inc. Thieme Publishers.

Holt, M.C. i Dowell, C.R. (2011). Actor Vocal Training for the Habilitation of Speech in Adolescent Users of Cochlear Implants. Journal of Deaf Studies and Deaf Education 16(1):140-51. DOI: 10.1093/deafed/enq033

Kral, A. (2013). To hear or not to hear: Neuroscience of deafness. In: Kral, A. Popper, N. A. Fay, R. R. Deafness (2013). New York: Springer.

Lachs, L, Pisoni, DB, \& Kirk, KI. (2001). Use of audiovisual information in speech perception by prelingually deaf children with cochlear implants: A first report. Ear and Hearing, 22, pp. 236-251.

Pisoni, B.D. Speech Perception in Deaf Children with Cochlear Implants (2004). Retrived from: http://www.rle.mit.edu/ soundtosense/conference/pdfs/invitedspeakers/Pisoni\%20 PAPER.pdf, pp. 133-147

Speechreading. (2016). U Supporting success for children with special needs. Retrived from: https://successforkidswithhearingloss.com/for-professionals/speechreading/

Spencer, P. E. (2016). It seems like only yesterday. In: Marshark, M. Spencer, P. E. (2016). The Oxford handbook of deaf studies in language. New York: Oxford Universiy Press. 